\title{
UN PROGRAMA NACIONAL DE ACCESO A LA INFORMACIÓN CIENTÍFICA
}

Anna María Prat* 


\section{RESUMEN}

Este artículo expone, en primer lugar, las importantes transformaciones que acontecen en la actualidad en la comunicación científica, derivadas del uso creciente de las publicaciones electrónicas y de las nuevas tecnologías de información. En segundo término, explora las posibilidades y dificultades que los científicos y el público en general tienen de acceder a la información científica. Finalmente, aporta antecedentes acerca de los variados programas que, en este ámbito, se llevan a cabo en Chile.

\section{ABSTRACT}

To begin with, this report shows nowadays' important changes regarding scientific communication due to the easier access to electronic papers and to the new electronic information. Secondly, it explores the possibilities as well as the difficulties that scholars and common public have to face in order to obtain scientific information. Finally, it talks about the different programs that take place in Chile. 


\section{UN PROGRAMA NACIONAL DE ACCESO A LA INFORMACIÓN CIENTÍFICA}

\section{ANTECEDENTES}

En la actualidad, y en todo el mundo, se viven acalorados debates respecto a la evolución y validez de los sistemas tradicionales de comunicación científica y de la publicación y difusión de trabajos académicos. Estos debates se centran, preferentemente, en los costos crecientes de la publicación y edición de revistas y textos, en las posibilidades que abren las nuevas tecnologías de información, en la validez de las formas tradicionales de evaluación de la producción científica y en la rapidez con que las instituciones modifican sus bibliotecas e infraestructuras de información para adaptarse a las nuevas formas y situaciones. Surgen diferentes propuestas y experimentos y, por encima de todo, los usuarios exigen, más que nunca, un acceso rápido, confiable y exhaustivo a la información existente. Esta situación hace imprescindible y urgente la definición de políticas nacionales y la implementación de programas tendientes a asegurar un acceso equitativo a la totalidad de la información que requiere el sistema científico y académico chileno.

Previo a describir el programa que estamos diseñando para Chile, parece interesante analizar algunas de las nuevas situaciones que influencian dichas políticas.

Desde que, en 1655, apareciera el primer número de Le Journal des Savants, la comunicación científica no ha sufrido cambios radicales. Como entonces, se realiza, primordialmente, a través de revistas. Esta forma, que reemplazó con éxito a la correspondencia privada, ha servido desde esa época para informar resultados, someter ideas y 
hallazgos al juicio de los pares, asegurar la prioridad del descubrimiento y acumular conocimiento para usos futuros.

Como dice Joost Kircz', el "objetivo de la comunicación científica es el registro, evaluación, diseminación y acumulación del conocimiento, hechos y percepciones humanas". Para estos efectos, y a lo largo del tiempo, la comunidad científica ha desarrollado un complejo sistema de relaciones y organizaciones que le han permitido producir, estructurar y validar la base de conocimiento existente.

Tal como lo definiera Kuhn² a mediados de los años '60, las comunidades científicas se organizan alrededor de disciplinas que comparten un conjunto heterogéneo de conceptos, teorías, métodos e instrumentos, experiencias, formas de aprendizaje, costumbres y reglas de trabajo, que nos permiten distinguir e identificar una comunidad específica en cualquier parte del mundo en que se encuentre. Participan de los mismos intereses y métodos de investigación, y se mantienen y perpetúan a través de la formación de nuevos investigadores, la generación y utilización de la misma literatura científica, etc. Son grupos que comparten el lenguaje de una disciplina específica, que difícilmente tienen en común los paradigmas de otros grupos o comunidades científicas, pero que, sin embargo, al utilizar un modelo de pensamiento racional, como es el método científico, configuran una comunidad nacional e internacional que se reconoce a si misma y es reconocida como tal.

Esta forma de trabajo, generalmente desarrollada en instituciones académicas, es lo que Gibbons et. al., ${ }^{3,4}$ definen como Modo 1, en contraposición al Modo 2 de hacer ciencia que empieza a surgir como una ruptura con las antiguas estructuras centradas en una disciplina. En el Modo 2, el conocimiento se produce para resolver un problema

1 Kircz,Joost, Scientific Communication as an object of science, http://www.science.uva.nl/projects/ commphys/papers/aceur.htm

2 Kuhn, Thomas, The Structure of Scientific Revolutions, University of Chicago Press, Chicago, 1962.

3 Gibbons, Michael, Limoges, Camile, Nowotny, Helga, Schwartzman, Simon, Scott, Peter and Trow, Martin, The New production of Knowledge. The Dynamics of Science and Research in Contemporary Societies, Sage, London, 1994.

4 Nowotny, Helga, Scott, Peter, Gibbons. Michel, Re-Thinking Science: Knowledge and the Public in an age of uncertainty, Polity Press and Blackwell Publishers, London, 2001 
preciso y será útil para alguien: industria, gobierno o, finalmente, la sociedad, y no se origina si no hay nadie interesado en él. Los grupos de trabajo se forman para este fin específico, son transdisciplinarios, aportan sus técnicas y métodos, transfieren conocimiento que se incorpora a una nueva tecnología, y no necesariamente se mantienen una vez superado el problema en estudio. La investigación se produce en una diversidad de lugares, desde institutos de investigación, laboratorios industriales, hasta organismos de gobierno o consultoras, interactuando entre sí. En esta nueva forma de hacer ciencia, se crean redes, formales e informales, que se comunican de formas diversas, usando métodos electrónicos, organizacionales o sociales. La interacción de los diferentes sitios donde se investiga, en la que participan grupos cada vez más especializados, genera con el tiempo nuevos subcampos. Su producción se aparta así cada vez más de la forma disciplinaria y académica tradicional.

Esta nueva modalidad tiene también implicancias importantes en el control de calidad. En el Modo 1, éste se ejerce a través de la opinión de pares sobre las contribuciones de individuos En el Modo 2, en cambio, se le agregan nuevos criterios, tales como: si la solución encontrada será competitiva en el mercado, si el costo será efectivo o si será socialmente aceptable. La calidad no es responsabilidad sólo de un grupo de pares de una misma disciplina. El abanico de criterios es mucho más amplio y debe asegurar que se está haciendo buena ciencia además de ciencia útil.

Otro de los cambios que se está produciendo en este ámbito es la necesidad de responder a la sociedad (que, finalmente, es quien financia los programas de investigación) acerca de los resultados de los programas y proyectos que se están desarrollando. Cuando la ciencia se hacía, se comunicaba y se transmitía en el plano puramente académico y sólo se debía responder frente a los pares, las formas de comunicación eran precisas, normadas y limitadas a publicaciones altamente especializadas. Hoy, se supone que el conocimiento producido debe estar disponible para que la sociedad esté informada. Sin perder la rigurosidad científica, muchas revistas electrónicas publican actualmente secciones abiertas donde los resultados de la investigación pueden ser conocidos, juzgados y aceptados o rechazados por el público. Esto es un cambio profundo frente a lo que estábamos 
acostumbrados respecto de la ciencia, de sus lenguajes y sus convenciones que parecían tan esotéricas para el ciudadano común.

Estos cambios en la percepción y en la forma de hacer ciencia influyen profundamente en cómo se comunican sus hallazgos. Se transforma la tradicional cadena lineal de comunicación de información en una red interactiva de transmisión de resultados, con reglas y normas propias que están cambiando de manera profunda todas las formas de transmitir, evaluar y acumular conocimiento ${ }^{5}$. Incluso un aspecto que no se había puesto en duda hasta ahora, el concepto de evaluación por pares, está siendo cuestionado. En el mes de febrero recién pasado, la Royal Society ${ }^{6}$ anunció la creación de un comité para explorar nuevas formas alternativas de evaluación para mejorar el sistema existente de validación por pares y devolverle la credibilidad a la ciencia frente al público, ya que todo el proceso ha dejado de ser confiable. Se espera que el comité produzca dos tipos de documentos: una guía para mejorar las prácticas de la comunicación científica y un "Science Brief" destinado al público, que le permita interpretar mejor los resultados de la investigación científica. Esto se agrega al informe publicado días antes por la Colaboración Cochrane ${ }^{7}$ acerca de la revisión editorial por pares destinada a mejorar la calidad de los estudios biomédicos, en el que se concluye que hay poca evidencia que permita demostrar que la evaluación por pares asegura buena ciencia o, al menos, buena ciencia en el área de la salud. Todo esto remece, indudablemente, una parte muy importante del sistema en el que se ha basado la comunicación científica hasta hoy.

Como ya se ha dicho, las funciones de ésta son y han sido: el registro de nuevo conocimiento, la información y comunicación del mismo, la certificación de lo que se informa y su almacenamiento. Desde el siglo XVII se puso en marcha toda una mecánica de

5 Roosendaal, Hans E., Geurts, Peter A. Th.M., Forces and Functions in Scientific Communication: an analysis of their interplay, CRISP97: Cooperative Research Information_Systems in Physics. http://www.physik.uni-oldenburg.de/conferences/crisp97/roosendaal

6 Hagan, Pat, Peer review under scrutiny. Royal Society reacts to fears that the public has lost trust in science, en The Scientist, Daily News, 2003, February 3 http://www.biomedcentral.com/ news/20030203/4

7 Hagan, Pat, Review queries usefulness of peer review. Cochrane Review finds little evidence that peer-review ensures quality of biomedical research, en The Scientist, Daily News, 2003, January 28 http://www.biomedcentral.com/news/20030128/5 
comunicación, certificación y acumulación de conocimiento. A lo largo de los años, todo el proceso se fue normalizando. Se crearon estructuras precisas para la comunicación de hipótesis, observaciones y resultados, se normalizaron las publicaciones y, al final, la comunidad científica terminó entregando todo el proceso de edición, publicación y distribución de sus revistas especializadas a agentes externos comerciales (las editoriales científicas), quienes dominan hoy el campo y se convirtieron -desde hace bastante tiempo-, en los guardianes y, a veces, cancerberos de la ciencia. Es curioso que en este proceso el científico, que es el principal protagonista -ya que es productor, consumidor y evaluador de la información-, haya llegado a tener tan poca injerencia en la gestión total del sistema.

Esta situación ha producido malestar y rebeliones. Todo el proceso está hoy en discusión. El primer grito de independencia surgió en el campo de la física, a partir de 1991, cuando estuvo disponible la tecnología necesaria para cambiar el sistema sin amenazar la calidad científica ni la integridad de la información que se comunicaba. La historia del primer archivo de preimpresos es ampliamente conocida y no es ésta la oportunidad para analizarla con detalle, sin embargo vale la pena resaltar algunas de sus características. El sistema se fundamenta en que es el autor quien archiva sus propios artículos, en que son los propios científicos quienes tienen el control de todo el proceso, que es lo suficientemente flexible como para convivir con las demás formas tradicionales de comunicación científica o adaptarse a las emergentes. El método permite una rápida diseminación de la información, y la evaluación del contenido se produce en forma simultánea o a posteriori y por la misma comunidad que lo mantiene. La información es gratuita y las capacidades de archivo que presenta son muy amplias. La creación de redes globales de conocimiento es una posibilidad cierta y atractiva. Es indudable que el sistema ha modificado profundamente la forma de comunicación en física, matemáticas, astronomía y ciencias afines, y sus posibilidades están afectando a la totalidad de las disciplinas y a las nuevas formas de comunicar, evaluar y acumular ciencia.

Las revistas científicas son todavía una realidad y, para muchos, que no cambiará en el corto plazo. La actual cadena de comunicación de la ciencia a través de publicaciones convencionales (electrónicas o 
en papel) está formada por una amplia gama de personas e instituciones, cada una con responsabilidades diversas. El sistema completo ha alcanzado costos que, al sumarse, alcanzan cifras que pocas instituciones, en forma aislada, pueden hoy asumir. Estos incluyen a los autores y a la producción del conocimiento, a los editores comerciales a cargo del proceso de evaluación por pares y de edición y distribución, y a las bibliotecas donde esta información se adquiere y almacena para usarse hoy y en el futuro. A juicio de Odlyzko ${ }^{8,9}$, este proceso, que pasa por tantos intermediarios, es caro y, a la postre, termina siendo poco eficiente. Las revistas en papel tienen cada vez tirajes menores y los costos, en consecuencia, sufren incrementos significativos todos los años. Las versiones electrónicas no han disminuido sus precios. En esta cadena terminan pagando el precio del acceso a los resultados de la investigación quienes han financiado su producción y lo hacen a precios cada día más exorbitantes. La pugna entre quienes mantienen el sistema y quienes desean quebrarlo para ampliar las posibilidades de acceso que las nuevas tecnologías permiten, no ha hecho sino empezar.

Uno entre muchos de los ejemplos de búsqueda de soluciones, es la iniciativa de un grupo de científicos de crear la Biblioteca Pública de la Ciencia, basada en el concepto de "open access" ya aplicado por un buen número de revistas científicas electrónicas en diversas disciplinas donde los costos son asumidos por los autores y, por lo tanto, pueden ser cargados a los gastos de los proyectos de investigación asumidos por organismos gubernamentales o privados que, de alguna manera, hoy deben asumir también los costos de acceso a precios cada vez mayores.

La propuesta de "open access" permite que el nuevo conocimiento generado sea público y gratuito, tanto para la comunidad científica como para la sociedad en general. La Budapest Open Access Initiative ${ }^{10}$, suscrita en diciembre del 2001, que busca, justamente, el acceso abierto

8 Odlyzko, Andrew, Competition and Cooperation: Libraries and Publishers in the Transition to Electronic Scholarly Journals, en The Journal of Electronic Publishing, 1999, Vol. 4 No. 4 http://www.press.umich.edu/jep/04-04/odlyzko0404.html

9 Odlyzko, Andrew, The Economics of Electronic Journals. en The Journal of Electronic Publishing,_1998, Vol. 4 No. 1 http://www.press.umich.edu/jep/04-01/odlyzko.html

10 Budapest Open Access Initiative. http://www.soros.org/openaccess 
y gratuito a la literatura científica revisada por pares, propicia dos caminos: los autoarchivos -o archivos de preimpresos ya mencionadosy una nueva generación de revistas de acceso abierto. Tal como señala el texto de la iniciativa, poner en marcha estos programas depende sólo de la voluntad de los científicos, no se deben esperar cambios en la legislación, en la metodología de trabajo o en las demandas del mercado. El grupo no sólo apoya estos sistemas de trabajo sino que propicia la investigación de nuevas formas que permitan la transición de las actuales modalidades de diseminación hacia el acceso abierto. Se buscan proyectos flexibles, innovadores y adaptados a las circunstancias locales.

Otra forma de encarar el aumento permanente de los costos de las publicaciones científicas, ha surgido, en todo el mundo, desde el ámbito de los especialistas en información y de los bibliotecarios, al crear consorcios para la adquisición cooperativa de publicaciones periódicas o acceso a la información digital. Estos consorcios están enfocando en forma integral todos los aspectos de la gestión de información: uso de enlaces de textos y de recursos, uso de los textos completos asociados a bases de datos especializadas, racionalización de colecciones, definición de "colecciones núcleo" indispensables en soporte físico en el país, entre otros. Esto ha permitido asegurar un uso equitativo y distribuido de la información por parte de todos los usuarios de un país o una región, a precios asequibles. Los modelos de consorcios son muchos y cambian de acuerdo con las características de cada grupo de instituciones.

En todo caso, las nuevas tecnologías de información y comunicación han cambiado la estructura misma del artículo formal tradicional. Esto también afecta a los sistemas de información que se están generando. Cuando surgió la posibilidad de publicar en forma electrónica, basándose en la tecnología web, muchos editores vieron en ésta la posibilidad de replicar las revistas tradicionales en papel, ya que el nuevo formato permitía un acceso más fácil, abarataba los costos de impresión y distribución, y permitía aprovechar las facilidades de la comunicación electrónica. Las primeras revistas electrónicas fueron clones de las revistas de papel, tanto las que se crearon especialmente para ese medio, como las que iniciaron ediciones paralelas al papel en formato electrónico. Sin embargo, al poco tiempo, las facilidades de edición del nuevo medio empezaron a cambiar y a acelerar la transformación de la revista tradicional. 
Por de pronto, los textos ya no debían ser lineales. Con la tecnología web, los textos pasaban a ser hipertextuales. Este cambio muy pronto tuvo importantes consecuencias. La posibilidad de combinar los textos con motores de búsqueda ofrece nuevas capacidades de almacenamiento y recuperación de información. Además las publicaciones pueden incluir materiales multimediales y no solamente textuales. La fotografía digital permite en muchas áreas del conocimiento una calidad difícilmente alcanzable en la versión impresa.

Asimismo, la posibilidad de agregar otro tipo de material audiovisual (videos o música) es importante y rápidamente utilizado en ciertas disciplinas. También la nueva tecnología termina, en cierta medida, con la restricción del espacio. En muchas áreas experimentales es importante agregar grandes conjuntos de datos y otros materiales que difícilmente podrían publicarse en revistas de papel, siempre restringidas respecto al número de páginas. Las características del nuevo medio posibilitan la comunicación con los autores y abren áreas interactivas, en las que pueden responder consultas y plantear debates sobre temas de interés. Las nuevas secciones destinadas a informar al gran público y divulgar hallazgos de la ciencia, así como la posibilidad de publicar los artículos una vez aprobados para su inclusión en la revista sin esperar la fecha de cierre de una edición, son algunos de los aportes de la nueva tecnología que han sido aprovechados por casi todas las publicaciones importantes.

Pero la verdadera innovación es la posibilidad de crear enlaces. Éstos se han convertido en importantes unidades de información en sí mismos y, muy pronto, necesitaremos analizar los sistemas de enlaces de nuestras publicaciones para conocer su uso e impacto, la pertenencia a redes o la participación en programas y proyectos internacionales de nuestros investigadores y sus equipos de trabajo.

Algunos de los tipos de vínculos más comunes son actualmente: de las referencias a los textos completos, como en el caso, entre otros, de CrossRef ${ }^{11}$ : el esfuerzo cooperativo de un grupo de editores que, al aplicar el sistema de Identificadores de Objetos Digitales (DOI), permite enlazar las referencias de los artículos de más de doscientos editores a

11 Crossref, http://www.crossref.org 
los textos completos y enlaces con los metadatos de editores secundarios tales como Medline o Web of Science. Otro tipo posible de enlaces es con bancos de datos científicos (cristalográficos, genéticos, químicos), con otro tipo de publicaciones a texto completo (tesis, patentes, literatura gris) y con bases de datos complementarias (currículos e instituciones). Se han iniciado experiencias muy interesantes, como la de vincular entre sí porciones de artículos y documentos que permitan la generación de nuevos conjuntos de información de acuerdo con requerimientos específicos. Esta malla de conexiones está dando como resultado una red global con diferentes nodos que abarcan la totalidad de la base de conocimiento existente. Frente a esta maraña de información, la American Association for the Advancement of Science, en conjunto con el Consejo Internacional de la Ciencia, ICSU y la UNESCO organizaron, en 1999, una reunión destinada a fijar normas para las nuevas formas de comunicación científica electrónica $^{12}$. La primera preocupación que se plantea en el documento final es la adecuada definición de lo que es una publicación científica. Se estima necesaria esta aclaración dadas las múltiples formas de publicar resultados de investigaciones en Internet y la dificultad de descubrir, a primera vista, las que son confiables.

Para calificar como tal se propone que un documento tenga las siguientes cualidades: 1) quedar fijado, esto es registrado en algún tipo de medio durable; 2 ) estar, en principio, públicamente disponible (no necesariamente en forma gratuita) y 3 ) tener permanencia, o sea, mantenerse en la misma forma y en el mismo lugar, de modo tal que pueda ser fácilmente accesible y recuperable a lo largo del tiempo. Además, se proponen cuatro características adicionales: debe garantizarse la autenticidad, esto es, que las diferentes versiones deben ser certificadas como auténticas y protegidas de cambios después de su publicación; debe asignársele una dirección o localización web permanente que identifique el documento sin ambigüedades; debe agregársele un registro bibliográfico (metadatos) público y libremente accesible para cualquier dirección y ubicación que describa el trabajo y sus diferentes versiones y debe haber un compromiso con el acceso público y con la posibilidad de recuperación a través de diferentes

12 Defining and Certifying Electronic Publication in Science, A proposal to the International Association of STM Publishers, October 1999, http://users.ox.ac.uk/ icsuinfo/aaas-stm.htm 
formas de archivo y preservación permanentes. Finalmente, debe asegurarse la calidad de lo comunicado para certificar su utilidad científica máxima y permitir alcanzar un alto nivel de confianza entre los lectores. Muchos de los programas que se han desarrollado después de ésta y otras reuniones internacionales para estos mismos efectos, se están ajustando a estas recomendaciones.

\section{EL PROGRAMA NACIONAL}

El Programa Nacional de Información Científica, definido el 2001, tomó en cuenta todas estas consideraciones, además de la necesidad de racionalizar el acceso a la información del sistema chileno de ciencia y tecnología, generar indicadores confiables y mejorar todo el proceso de postulación y gestión de los fondos concursables en línea. El Programa se concibió en tres módulos relacionados entre sí (produciendo una red de información integrada) que responden a diferentes requerimientos de información: CINCEL, el Consorcio para el Acceso a la Información Científica Electrónica, destinado a asegurar el uso del conocimiento generado en el mundo y que el país requiere para llevar a cabo sus programas de investigación y desarrollo; SciELO (Scientific Electronic Library on Line) destinado a asegurar la visibilidad de la información de alta calidad producida y publicada en Chile y SICTI el Sistema de Información en Ciencia Tecnología e Innovación, para asegurar el acceso a la información del sistema nacional de ciencia y tecnología. Los tres programas, íntimamente relacionados entre sí, debían utilizar las nuevas tecnologías disponibles y tomar en cuenta las recientes tendencias tanto de la comunicación como de la gestión de información.

\section{CINCEL. Consorcio para la Información Científica Electrónica}

Para asegurar el uso equitativo de la información científica y tecnológica internacional se creó, en el año 2002, un Consorcio en el que participan, además de CONICYT, las 25 universidades que reciben financiamiento por parte del Estado y pertenecen al Consejo de Rectores. Se está completando la tramitación de su personería jurídica y, en poco tiempo más, se iniciará la incorporación de universidades privadas y organismos de investigación públicos y privados. 
El principal objetivo de CINCEL es la adquisición cooperativa de acceso a la información científica internacional, especialmente en formato electrónico. Una primera etapa ha sido la suscripción y adquisición de información retrospectiva del Web of Science de ISI, servicio que, por su alto costo, ninguna institución podía suscribir por sí sola. Para el pago de la suscripción anual se acordó, entre todos los miembros, una tabla con aportes diferenciados de cada una de las instituciones, calculada de acuerdo con el uso potencial que cada una puede hacer del sistema. Para ello se ha tomado en cuenta el número de investigadores, los proyectos de investigación financiados a través de fondos concursables, número de posgrados, académicos equivalente jornada completa y, finalmente, la matrícula total. Esto permitió definir cinco tramos: quienes pueden, potencialmente, usar más el sistema pagan una cuota más alta, pero todos los miembros tienen el mismo acceso, por tiempo indefinido, durante todos los días del año desde cualquier computador conectado a la red de la universidad. Gracias a un proyecto financiado por Fundación Andes fue posible ampliar la cobertura del Web of Science a las áreas de Ciencias Sociales como también Artes y Humanidades y, con aportes de un proyecto Mecesup, el financiamiento de quince años de información retrospectiva.

En estos momentos se está estudiando la segunda fase del programa que incluye, entre otras, las siguientes actividades: acuerdo acerca de una política nacional de suscripción a publicaciones en papel que asegure que en el país se mantendrá, al menos, una colección física de las publicaciones consideradas indispensables; negociación de adquisición cooperativa de acceso a los textos completos, en formato electrónico, empezando, probablemente, por algunas áreas prioritarias que permitan evaluar el sistema; adquisición de accesos a bases de datos secundarias en ciertas áreas del conocimiento de alta demanda. Se han recibido solicitudes de varias universidades para incorporar al sistema, la suscripción a bases de resúmenes o de textos completos especializadas; creación de un fondo común que permita y estimule la publicación de trabajos científicos nacionales en revistas acogidas al sistema de "Open Access"; preparación de un portal de acceso a la información disponible, tanto en forma pagada como gratuita, por disciplinas; creación de un fondo común para el acceso, especialmente de las universidades más pequeñas, a textos completos bajo la 
modalidad de "pago por uso". Muchas de estas actividades están ya siendo desarrolladas por las universidades más grandes, pero es importante asegurar a todos un acceso igualitario a costos razonables. Se está trabajando igualmente con las asociaciones científicas, nacionales e internacionales, en la validación de nuevas formas de edición y en la negociación colectiva con editores especializados, que permitan un uso fluido de la información de cada una de las diferentes disciplinas.

De acuerdo con los estatutos de CINCEL, pueden integrarse al consorcio, además de las universidades públicas o privadas, los institutos de investigación y organismos gubernamentales, o empresas que realicen investigación.

SciELO, Scientific Electronic Library on Line

Cuando, en 1997, CONICYT inició la aventura de crear un programa de edición electrónica, lo que se pretendía era aprovechar las posibilidades que brindaba la nueva tecnología para darle visibilidad a una parte importante de la investigación que se llevaba a cabo en el país y que nunca sería publicada en las grandes revistas internacionales. Esto es especialmente válido en ciertas áreas del conocimiento. Publicar en español y en revistas locales es una práctica que se lleva a cabo con mucho esfuerzo desde hace muchos años y que deseábamos fomentar en diversas disciplinas. Las publicaciones locales no sólo cumplen con su papel tradicional de comunicar resultados científicos, también son un apoyo para la docencia y un sistema importante de comunicación con los usuarios potenciales de los resultados obtenidos. El programa tenía dos vertientes: un proyecto piloto para crear una revista científica cien por cien electrónica desde sus inicios -con el mayor rigor científico posible- y la transformación de las mejores revistas científicas nacionales de su formato en papel a un formato electrónico.

El proyecto de creación de una nueva revista ha sido muchas veces relatado. Se invitó a diferentes instituciones a participar. Respondió la Universidad Católica de Valparaíso y el Comité Chileno de Biotecnología y se creó, en 1998, la revista Electronic Journal of 
Biotechnology que, después de estos años, ha logrado un gran impacto: mantener una alta calidad de sus artículos (un 30\% de rechazo), introducir y experimentar con innovaciones de interés, tales como aceptar materiales no textuales, agregar secciones especiales con actas de congresos internacionales, resúmenes de los artículos -escritos por los mismos autores y destinados al público en general- y espacios interactivos, entre otros. La revista fue rápidamente incorporada a las principales bases de datos de resúmenes de las áreas afines y fue aceptada por ISI para ser incorporada a sus servicios. Para todos los efectos se considera que el experimento fue exitoso y se estudia la posibilidad de replicarlo en otras áreas del conocimiento.

La segunda parte del programa, la edición en formato electrónico de revistas tradicionales, se inició en el mismo año, adoptando la metodología SciELO desarrollada conjuntamente por el Centro Latinoamericano y del Caribe de Información en Ciencias de la Salud (BIREME/OPS) y la Fundación para la Investigación Científica, del Estado de Sao Paulo (FAPESP). Esto permitió participar, desde el comienzo, en una red de revistas latinoamericanas de alto nivel científico, que utiliza sistemas de evaluación similares y metodología compatible. Hoy SciELO-Chile cuenta ya con 39 títulos de revistas, a texto completo, asequibles en línea, la mayoría con colecciones a partir de 1997 y el número de títulos se sigue incrementando.

Dada la rigurosidad del sistema de evaluación, en el año 2002 las publicaciones incorporadas en SciELO Chile han sido aceptadas por el Ministerio de Educación para el cálculo del 5\% del Aporte Fiscal Directo a las universidades. Cabe destacar que, desde su inicio, tres de las revistas incorporadas a SciELO Chile han sido aceptadas para su inclusión en las bases de datos de ISI, lo que confirma la visibilidad alcanzada por las publicaciones nacionales.

A partir del año pasado, se inició la transferencia de la plataforma a algunas universidades para que editen sus propias publicaciones utilizando la misma metodología, y estamos en conversaciones con algunos ministerios, como el de Agricultura y el de Salud, para iniciar programas de edición temáticos enlazados con el sitio central SciELO. 
SICTI. Sistema de Información en Ciencia, Tecnología e Innovación ${ }^{13}$

El tercer módulo es el programa SICTI, inaugurado oficialmente en el mes de agosto del presente año. Financiado con recursos del Convenio Gobierno de Chile BID, en conjunto con aportes de la empresa privada, apunta a mejorar la competitividad científica y tecnológica del país mediante una infraestructura de información abierta, que facilite la interacción de la comunidad nacional e internacional en la identificación, desarrollo y gestión de procesos asociados a la investigación e innovación.

SICTI incluye información acerca del sistema de ciencia y tecnología nacional. Está compuesto por diferentes módulos y bases de datos: currículos, instituciones, proyectos y publicaciones, un sistema para generar indicadores y un portal para acceso a la información nacional. El programa permitirá, además, la gestión en línea de todo el proceso de postulación, evaluación, adjudicación y seguimiento de los proyectos y programas financiados por CONICYT. En el caso de los currículos se utilizará la plataforma Lattes desarrollada por el CNPq, de Brasil, la que será también utilizada por otros organismos nacionales de ciencia y tecnología de América Latina como parte del programa regional SciENTI, buscando crear una base de datos de investigadores íberoamericanos que permita, no sólo el intercambio de científicos, sino también la realización de trabajos conjuntos, el uso de expertise regional para evaluación de proyectos y programas, y la integración de redes regionales e internacionales.

El procedimiento permitirá compartir estándares, elaborar indicadores válidos para todo el Sistema Científico Nacional, acceder a currículos en línea de todos los investigadores chilenos y extranjeros y construir e implementar nuevas herramientas de formulación, evaluación y gestión de proyectos. Está concebido de forma tal que pueda ser transferido a universidades y otras instituciones interesadas, tanto en parte como en su totalidad.

Pero la importancia del Programa Nacional es su capacidad de interconexión. Ya en este momento, a partir de SciELO y de la unidad

13 Sistema de Información en Ciencia, Tecnología e Información, http://www.sicti.cl 
"artículo científico", se inicia una red de enlaces: de las referencias a diferentes bases de datos y, al menos, a los resúmenes de los documentos citados; de los autores a sus currículos en las bases de datos asociadas a SciENTI; enlaces entre artículos en los demás sitios SciELO a las bases de datos LILACS, MEDLINE y, muy pronto, con el Web of Science, entre otros. Cuando SICTI esté operativo, los enlaces, además, incluirán proyectos de investigación con su productividad, enlace con las bases de datos ISI, como, asimismo, con los textos completos suscritos a través de CINCEL. Esto permitirá descubrir una serie de relaciones entre instituciones y personas en Chile y la región, obtener datos de productividad e impacto nacional y regional e integrar nuestras publicaciones nacionales seleccionadas con la corriente mundial de información científica.

Creemos que la situación mundial en comunicación científica, así como las nuevas tecnologías, ofrecen una oportunidad única para innovar en este campo a medida que nuevas posibilidades surgen en el mundo. El estar lejos ya no es una barrera. La única barrera es hacer mal las cosas o producir ciencia mediocre. Esperamos que este no sea el caso. 
